\title{
Intermittent hypoxia in rat enhancing peritoneal membrane thickening through HIF-1 $\alpha$-induced cytokines in peritoneum
}

\author{
Wasin Manuprasert, ${ }^{1}$ Asada Leelahavanichkul, ${ }^{4}$ Sirigul Kanjanabuch, ${ }^{1}$ Preecha Ruangvejvorachai, ${ }^{2}$ \\ Krissanapong Manotham, ${ }^{6}$ Sompol Sanguanrungsirikul, ${ }^{3}$ Talerngsak Kanjanabuch ${ }^{1,5,7}$
}

\begin{abstract}
Background: Due to the high prevalence of both obstructive sleep apnea syndrome (OSA) and end-stage renal disease (ESRD), the co-existence of both conditions in peritoneal dialysis is demonstrated. Because OSA-induced chronic intermittent hypoxia is well-known, the hypoxia might worsen peritoneal membrane.

Objective: We tested the influence of chronic intermittent hypoxia upon peritoneal membrane in a Sprague-Dawley rat model.

Methods: Normal saline or 3.86\% glucose peritoneal dialysis fluid (PDF) were intra-peritoneally administered twice a day as negative (NSS group) and positive controls (PDF group), respectively. Intermittent hypoxia was induced by using a hypoxic chamber with $10 \% \mathrm{O}_{2}$ for 8 hours a day plus twice-daily NSS injection (IH group).

Results: At 12 weeks of the experiments, high serum TNF- $\alpha$ and IL-6 (but not IL-10) with normal renal and liver functions were demonstrated in the IH group (but not the PDF group). In parallel, local cytokines (TNF- $\alpha$, IL-6, and IL-10 in peritoneal membrane) and peritoneal membrane thickness were increased whereas peritoneal membrane hypoxia

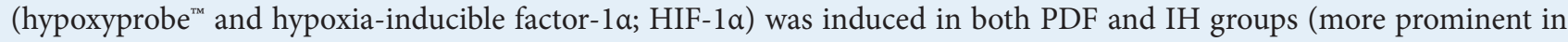
the PDF group). However, the increased vascular density in submesothelial area was established only in the PDF group.

Conclusion: Intermittent hypoxia model induced local peritoneal membrane inflammation and enhanced peritoneal membrane thickness, at least in part, through a mechanism of hypoxia-induced HIF-1a. Although peritoneal membrane alterations from PDF were more prominent than intermittent hypoxia, the combination between intermittent hypoxia with PDF utilization might facilitate peritoneal membrane failure, which will need more study.
\end{abstract}

Key words: Peritoneal dialysis, peritoneal membrane, sleep apnea syndrome, intermittent hypoxia, HIF-1 $\alpha$, peritoneal inflammation

\section{Citation:}

Manuprasert, W., Leelahavanichkul, A., Kanjanabuch, S.,

Ruangvejvorachai, P., Manotham, K., Sanguanrungsirikul, S.,

Kanjanabuch, T. (2022). Intermittent hypoxia in rat enhanc-

ing peritoneal membrane thickening through HIF-1 $\alpha$-induced

cytokines in peritoneum. Asian Pac J Allergy Immunol, 40(2),

178-186. https://doi.org/10.12932/ap-290519-0570

\footnotetext{
Affiliations:

${ }^{1}$ Center of Excellence in Kidney Metabolic Disorders,

${ }^{2}$ Department of Pathology,

${ }^{3}$ Department of Physiology,

${ }^{4}$ Department of Microbiology, and

Division of Nephrology, Department of Medicine, Faculty of Medi-

cine, Chulalongkorn University, Bangkok, Thailand
}

${ }^{6}$ Molecular and Cell Biology Unit, Department of Medicine, Lerdsin General Hospital, Bangkok, Thailand

7 PD Excellence Center, King Chulalongkorn Memorial Hospital, Bangkok, Thailand

Corresponding author:

1. Talerngsak Kanjanabuch Division of Nephrology, Department of Medicine, Faculty of Medicine, Chulalongkorn University, Bangkok 10330, Thailand

E-mail: golfnephro@hotmail.com

2. Asada Leelahavanichkul

Department of Microbiology, Faculty of Medicine, Chulalongkorn University, Bangkok 10330, Thailand E-mail: aleelahavanit@gmail.com 


\section{Introduction}

Obstructive sleep apnea (OSA), the most common sleep-related disorder world-wide, ${ }^{1}$ is the apnea-induced intermittent hypoxia associated with several aspects of renal disorder, including increased renin-angiotensin aldosterone system, enhanced sympathetic nervous system activities, facilitated reactive oxygen species (ROS), and induced focal segmental glomerulosclerosis-liked kidney characteristics. ${ }^{2-5}$ On the other hand, peritoneal dialysis (PD) is a live-saving therapy for patients with end-stage renal disease (ESRD) by removing the waste products and excess fluid from blood circulation into dwelt PD fluids using peritoneum as a semi-permeable membrane. High glucose concentration in PD fluids is necessary for driving excess fluid across peritoneal membrane called ultrafiltration. The peritoneal membrane alterations in long-term exposure to PD fluids, including peritoneal membrane thickening, peritoneal fibrosis, angiogenesis, and vascular obliteration are well documented. ${ }^{6}$ Because i) OSA is common in the population, ${ }^{1}$ ii) OSA enhances hypertension, cardiovascular disease, and renal injury, ${ }^{5,7}$ and iii) more than $60 \%$ of patients with $\mathrm{PD}$ report OSA ${ }^{8}$ then OSA might have some impacts upon PD.

Indeed, OSA associated with increased oxidative stress in several sites, systemically and locally; such as in brain and adipocyte. ${ }^{9-11}$ Then chronic inflammation from OSA induced intermittent hypoxia might enhance peritoneal membrane defect resulting in inadequate dialysis. Unfortunately, the data on the impact of OSA and intermittent hypoxia upon PD is still lacking. In addition, PD fluids induce through blood-vessel obliteration (or narrowing) and peritoneal hypoxia possibly through the induction of HIF-1a (a transcriptional factor responsible for hypoxic response) is well-known. ${ }^{12-15}$ Indeed, the transfection of HIF-1a in mice enhances peritoneal membrane alterations. ${ }^{16}$ Because the direct linkage between OSA and peritoneal membrane injury is never explored, then our objective is to examine the influence of chronic intermittent hypoxia upon the peritoneal membrane in comparison with chronic PD fluid exposure. We then explored the peritoneal membrane alterations in rat models of chronic intermittent hypoxia (a representative model of OSA) and chronic PD fluids (3.86\% glucose) administration.

\section{Materials and Methods \\ Animal model and histological analysis}

Animal study protocol number 4/2559 approved by the Institutional Animal Care and Use Committee at the Faculty of Medicine, Chulalongkorn University following National Institutes of Health (NIH) criteria was used. Male, 8 weeks old Sprague-Dawley rats (weights between 250-300 grams), purchased from National Laboratory Animal center (NLAC), Mahidol University, Thailand, were maintained with 12 hours/12 hours light cycle, fed ad libitum and subjected to twice-daily IP injection $(10 \mathrm{~mL} / 100 \mathrm{~g})$ with sterile normal saline (NSS group), 3.86\% glucose PD fluids (PDF group; Baxter Healthcare, Thailand) or sterile normal saline plus hypoxic induction by hypoxic chamber $\left(90 \% \mathrm{~N}_{2}, 10 \% \mathrm{O}_{2}\right.$ for 8 hours/day during $8 \mathrm{AM}-4 \mathrm{PM}$ ) which modified from Li C et al (IH group). ${ }^{17}$ All rats were sacrificed after 12 weeks of the experiments at 8 AM with cardiac puncture under isoflurane anesthesia. At sacrifice, samples from several sites, including parietal peritoneal membrane (peeling from abdominal wall), visceral peritoneal membrane (mesentery around cecum), kidney, and liver were snapping frozen with liquid nitrogen then stored at $-80^{\circ} \mathrm{C}$ until use. The abdominal wall and mesentery were also fixed with $10 \%$ neutral buffered formalin then embedded in the paraffin block for the Hematoxylin and Eosin (H\&E) and Masson's trichrome staining. The semi-quantitative analysis of histological staining with 5 different points per each image at $\times 200$ magnification using Image J program (NIH, USA) followed a published protocol was utilized. ${ }^{18}$

\section{Rat serum analysis and cytokines of internal organs}

Kidney functions (serum blood urea nitrogen and creatinine) were determined by QuantiChrom Urea assay (DIUR500, Bioassay, Hayward, CA, USA) and Creatinine assay (DICT-500, Bioassay), respectively. Liver function (serum alanine transaminase) and serum cytokines were evaluated by EnzyChrom Alanine Transaminase assay (EALT-100, BioAssay) and the enzyme-linked immunosorbent assay (ELISA) (Biolegend, San Diego, CA, USA), respectively. For tissue cytokine detection, tissue samples were weighed and sonicated thoroughly then the supernatant from homogenous tissue preparation was collected for cytokine measurement by ELISA assay (Biolegend) as mentioned earlier.

\section{The evaluation of hypoxia (blood vessel density and expres-

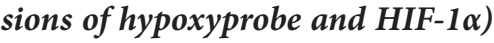

Because blood vessel density was associated with tissue oxygenation, blood vessel density in peritoneal membrane was evaluated after labelling with Lectin as a previous protocol. ${ }^{19}$ Briefly, $300 \mu \mathrm{g}$ of Lycopersicon esculentum Lectin (B-1175, Vector Laboratory, CA, USA) were administered into left cardiac ventricle at 5 minutes before euthanasia then the antigen retrieval agent (VECTASTAIN R.T.U. Elite ABC Reagent, PK7100, Vector Laboratory, CA, USA) was applied on de-paraffinized tissue section for 30 minutes at room temperature before enumeration of blood vessels number (diameter ranging from $10-80 \mu \mathrm{m}$ ) per $0.04 \mathrm{~mm}^{2}$.

Tissue hypoxia was assessed by staining with a 2-nitroimidazole hypoxia marker (hypoxyprobe ${ }^{\mathrm{Tm}}, \mathrm{HP1}-200$ kit, Hypoxyprobe, Inc., MA, USA) and visualized with immunohistochemical technique. In brief, $60 \mathrm{mg} / \mathrm{kg}$ of hypoxyprobe ${ }^{\mathrm{rm}}$ (HP1-200 kit, Hypoxyprobe, Inc., MA, USA) was IP administered for 30 minutes before euthanasia. Then, the immunohistochemistry analysis was performed using horseradish peroxidase (HRP)-3,3' diaminobenzidine (DAB) on paraffin-embedded peritoneal membrane tissue samples. After deparaffinization, $3 \mu \mathrm{m}$ of tissue sections were incubated with $3 \% \mathrm{H}_{2} \mathrm{O}_{2}$, anti-hypoxyprobe (HP1-200 kit, Hypoxyprobe, Inc., MA, USA.) and anti-mouse conjugated peroxidase (K4000, Dako, Glostrup, Denmark). Brownish color (positive staining) representing a localization of peroxidase activity was developed after adding DAB (K3468, Dako, Glostrup, Denmark). ${ }^{20}$ The staining is positive in the condition with tissue $\mathrm{pO}_{2}$ less than $10 \mathrm{mmHg}{ }^{21}$ Semi-quantitative analysis of hypoxic area with positive staining by the following score; $0=<1 \%, 1+=$ $1-5 \%, 2+=6-15 \%, 3+=16-30 \%$, and $4=>30 \% .{ }^{22}$ 
In addition, HIF-1a detection by Western blot analysis was used for hypoxia confirmation. Briefly, frozen peritoneal tissue was homogenized with radio-immunoprecipitation assay (RIPA) buffer (R0278, Sigma-Aldrich, MO, USA) and 50 $\mu \mathrm{g}$ of the protein was loaded on $10 \%$ SDS-PAGE then blot on PVDF membrane (Roche, Germany). Thereafter 5\% non-fat powdered milk (NB0669, Bio Basic Inc., NY, USA.) in PBS$0.1 \%$ Tween-20 was employed as a blocking agent before incubations with anti-HIF-1a (GTX 127309, GeneTex Inc., CA, USA) and anti- $\beta$-actin (\#4970, Cell Signaling Technology, MA, USA.). HIF-1 $\alpha$ positive control was available from Novus Biological $^{\mathrm{Tm}}$ (NB800-PC26, Novus Biological, CO, USA). The specific band was assessed by the chemiluminescent method using SuperSignal ${ }^{\mathrm{Tm}}$ West Femto Maximum Sensitivity Substrate kit (\#34096, Thermo Fisher Scientific, MA, USA). The images were recorded with ChemiDoc ${ }^{\mathrm{mm}}$ Touch Gel Imaging System (Bio-Rad, CA, USA) and the intensity of the protein bands was analyzed using Quantity One software (Bio-Rad). The level of HIF-1a protein expression was reported as a density ratio to $\beta$-actin. ${ }^{23}$

\section{Statistical analysis}

All data were expressed as mean \pm standard error (SE) and one-way analysis of variance (ANOVA) followed by Tukey's analysis was used for the comparisons of multiple groups. The chosen level of statistical significance was $p<0.05$.

\section{Results}

Intermittent hypoxia enhanced inflammation (locally and systemically) with normal internal organ functions

Despite hypoxia-induced several organ damages, the injury from intermittent hypoxia (IH group) or PD fluids administration (PDF group) after 12 weeks activation was not enough to induce dysfunctions of kidney and liver as determined by normal values of blood urea nitrogen, serum creatinine, and serum alanine transaminase (Figure 1A-C). However, the IH group, but not the PDF group, increased serum pro-inflammatory cytokines (TNF- $\alpha$ and IL-6) compared with the NSS group, without the elevation of an anti-inflammatory cytokine (serum IL-10) (Figure 1D-F). Interestingly, intermittent hypoxia enhanced all of the measured cytokines in collected organs including peritoneal membrane, liver, and kidney (Figure 2) in comparison with the NSS group, despite the normal kidney and liver functions (Figure 1A-C). Of note, PD fluids also increased cytokines in peritoneal membrane similarly to intermittent hypoxia but not in liver and kidney (Figure 2A-I) suggesting the more localized impact than intermittent hypoxia.
A

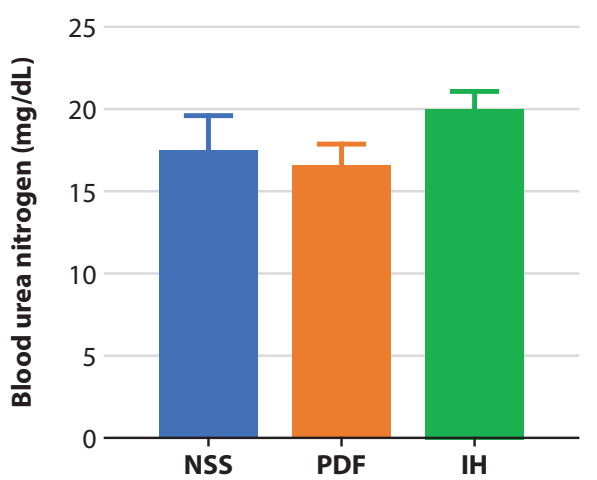

D

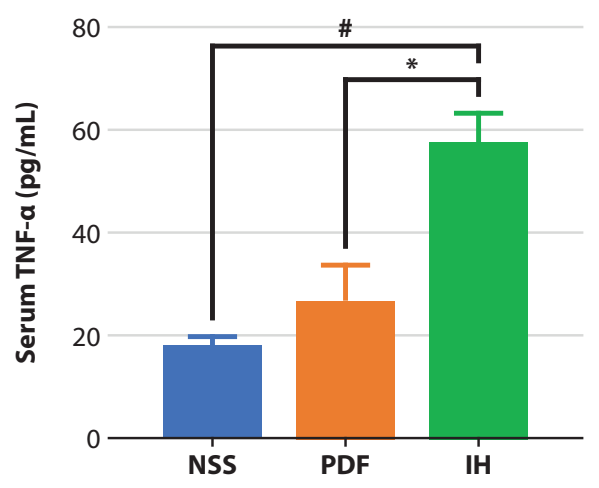

B

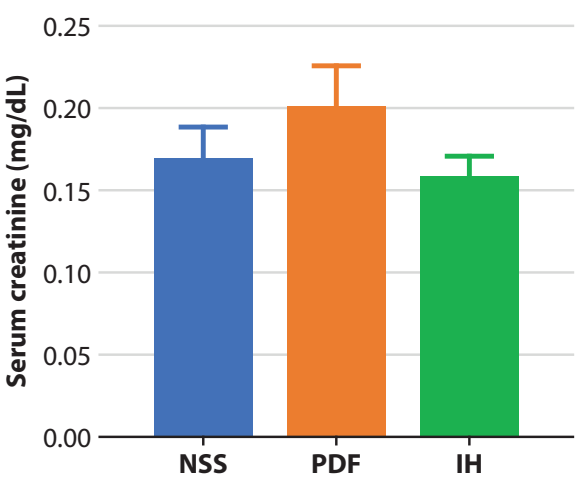

E



C

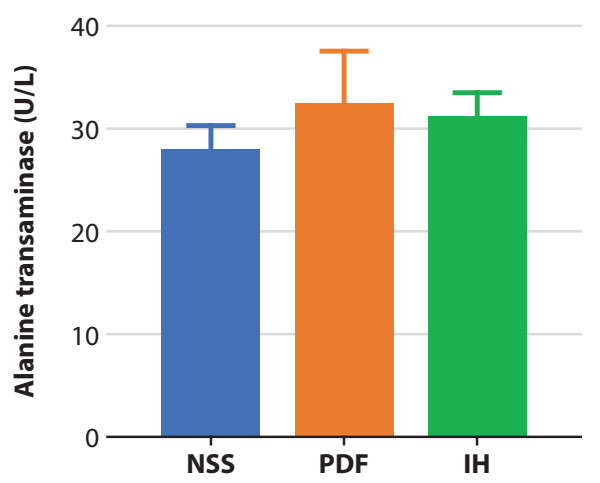

F

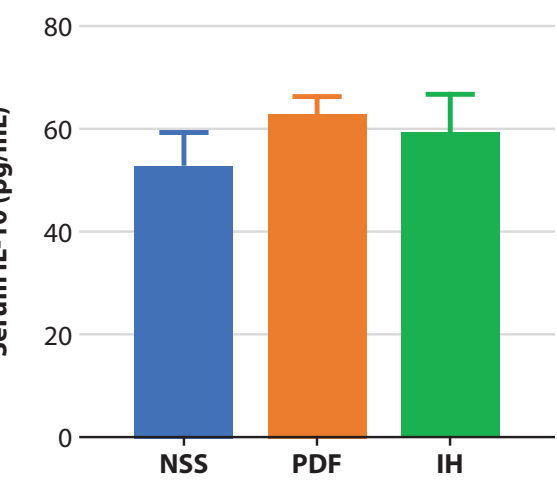

Figure 1. Renal injury as detemined by blood urea nitrogen (A), serum creatinine (B), and liver injury as evaluated by alanine transaminase (C) as well as serum cytokines (D-F) after 12 weeks administration of normal saline (NSS, negative control), peritoneal dialysis fluid (PDF, positive control), and NSS plus intermittent hypoxia (IH) were demonstrated (n = 5-6/ group). ${ }^{*} p<0.05$ value at IH vs. NSS, ${ }^{*} p<0.05$ value at IH vs. PDF 
A

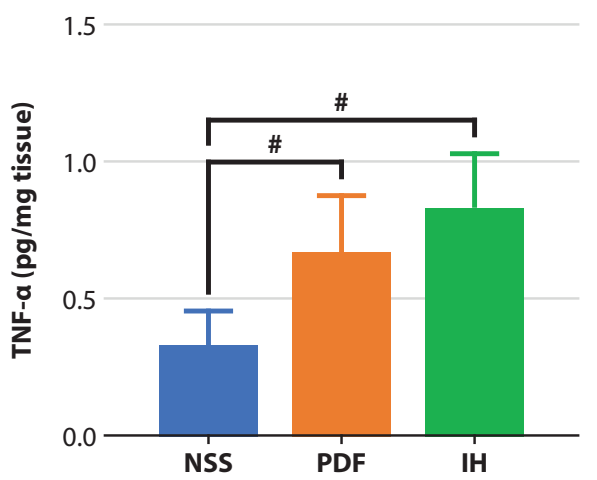

D

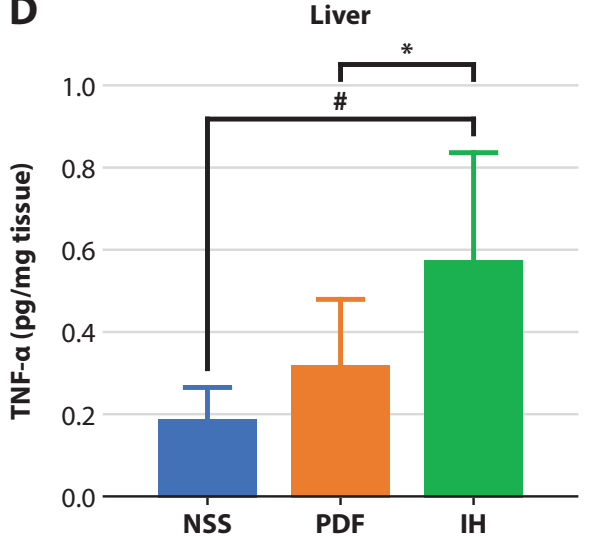

G

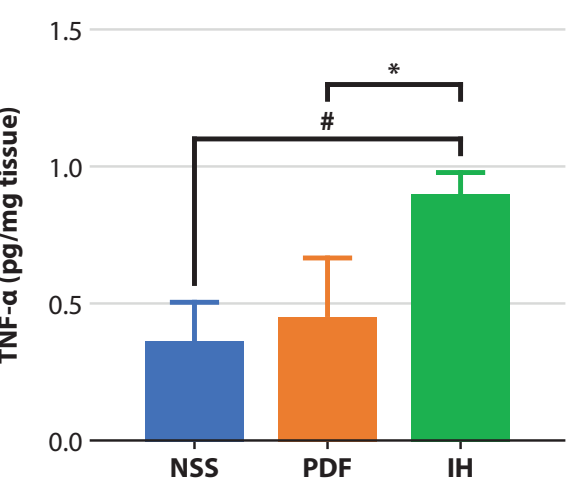

B

Peritoneum

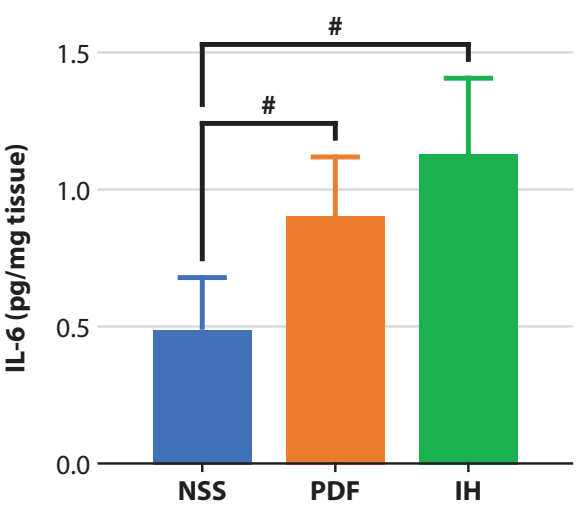

E



H

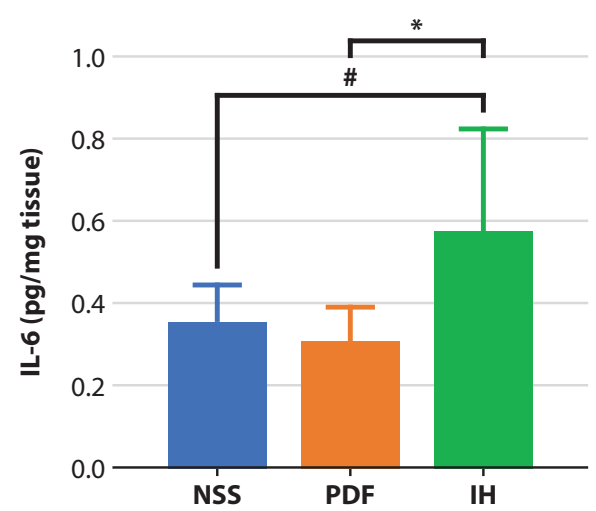

C

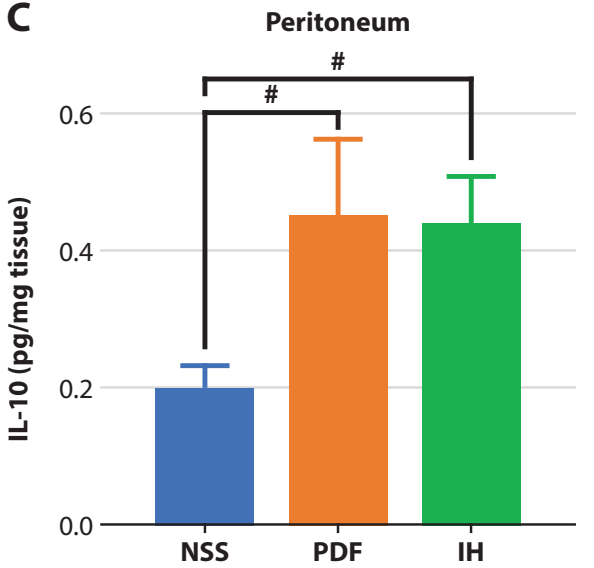

F

Liver

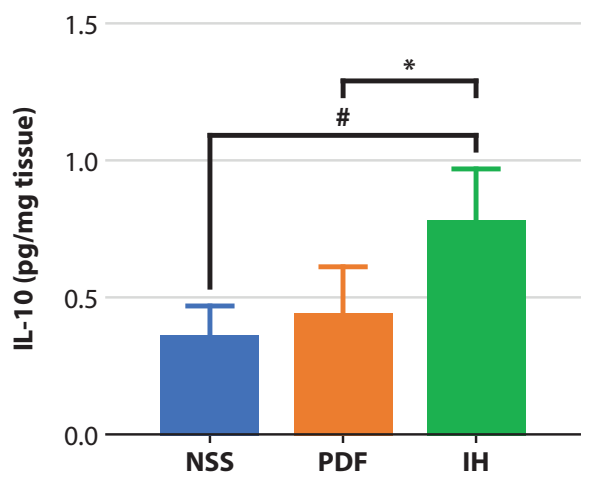

I

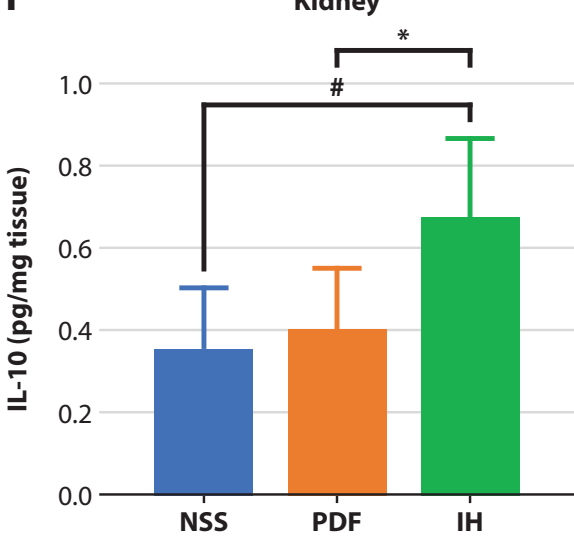

Figure 2. Local cytokines (TNF- $\alpha$, IL-6, and IL-10) from several sites at 12 weeks administration of normal saline (NSS, negative control), peritoneal dialysis fluid (PDF, positive control), and NSS plus intermittent hypoxia (IH) including parietal peritoneum (A-C), liver (D-F), and kidney (G-I) were demonstrated ( $n=5-6 /$ group). ${ }^{*} p<0.05$ value at experimental groups (PDF or IH) vs. NSS, ${ }^{*} p<0.05$ value at IH vs. PDF

Intermittent hypoxia enhanced peritoneal membrane thickening

With histological staining by H\&E and Masson's trichrome, peritoneal membrane thickness of PDF group was highest among 3 experimental groups (Figure 3) with several scatter inflammatory lesions in peritoneal membrane (Figure 3B, inset) supporting PD fluids-enhanced peritoneal membrane inflammation/alterations in the previous study. ${ }^{14}$ Interestingly, intermittent hypoxia also induced peritoneal membrane thickening; however, with a less extent compared to the PDF group (Figure 3). Of note, there were no inflammatory lesions in peritoneal membrane of the IH group (data not shown). 

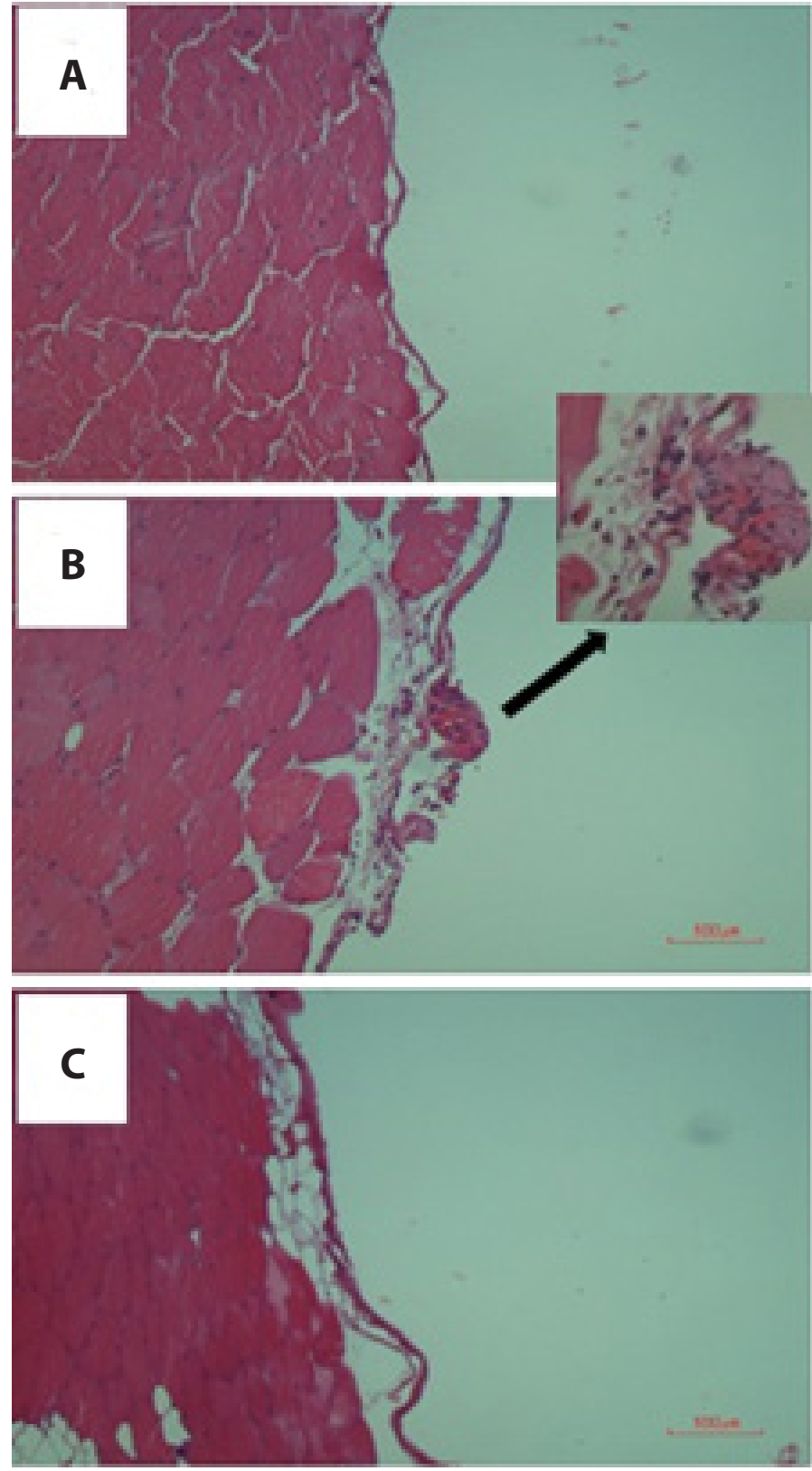

D

H \& E staining




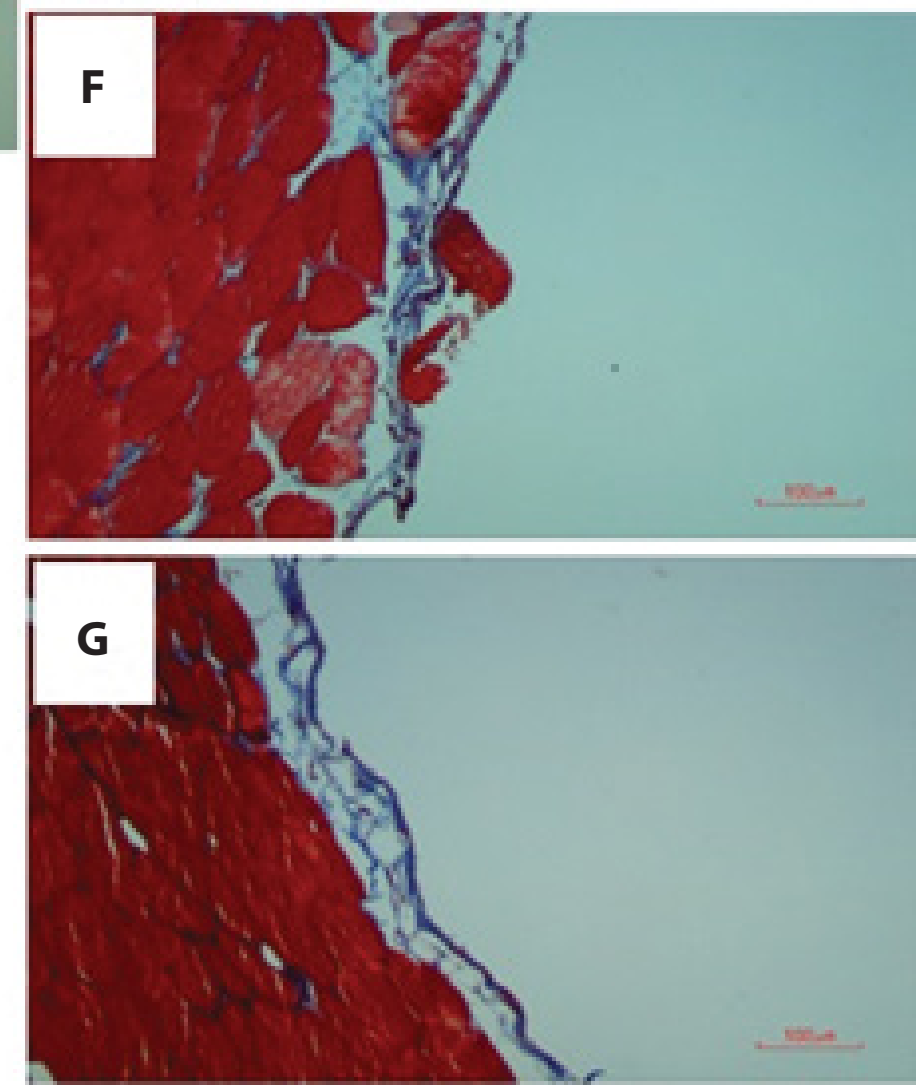

H

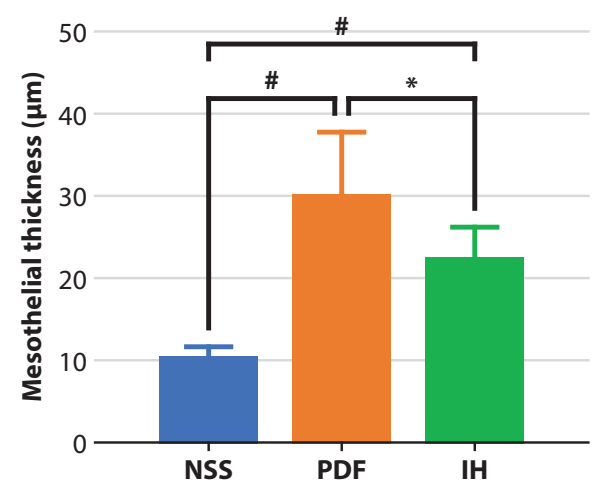

Figure 3. The representative figures of abdominal wall with $\mathrm{H} \& \mathrm{E}(\mathrm{A}-\mathrm{C})$ and Masson's trichrome stains (E-G) as well as the peritoneal membrane thickness $(\mathrm{D}$ and $\mathrm{H}$ ) at 12 weeks after administration of normal saline (NSS, negative control, A and E), peritoneal dialysis fluid (PDF, positive control, B and F) and NSS plus intermittent hypoxia (IH, C and G) were demonstrated $(\mathrm{n}=$ 5-6 for $\mathrm{D}$ and $\mathrm{H}$, the in-set figure representing 400x magnification of a scatter inflammatory lesion in PDF group). ${ }^{*} p<0.05$ value at experimental groups (PDF or IH) vs. NSS, ${ }^{*} p<0.05$ value at IH vs. PDF 
Intermittent hypoxia reduced blood vessels, enhanced hypoxia and induced HIF-1a

Blood vessel alteration in sub-mesothelial area is one of the mechanisms responsible for peritoneal membrane thickening. ${ }^{14}$ As such, PD fluids increased the density of vessels in this area, while hypoxia did not alter vascular density (Figure 4A-D). Indeed, the increased vascular density in the PDF group (Figure 4B) might be associated with the scatter inflammatory lesions in peritoneal membrane (Figure 3B). Among 3 experimental groups, the degree of hypoxia in peritoneal membrane was highest in the PDF group as determined by hypoxyprobe and HIF-1a burdens (Figure $4 \mathrm{E}-\mathrm{I}$ ) despite the increased vascular density only in the PDF group (Figure 4B) suggesting that insufficient blood flow or the relatively high energy requirement than the supplied oxygen perfusion of the PDF group. Although intermittent hypoxia did not alter vascular density (Figure 4C), intermittent hypoxia-induced hypoxia and activated HIF-1a in comparison with the NSS group (Figure $\mathbf{4 H}$ and 4I).
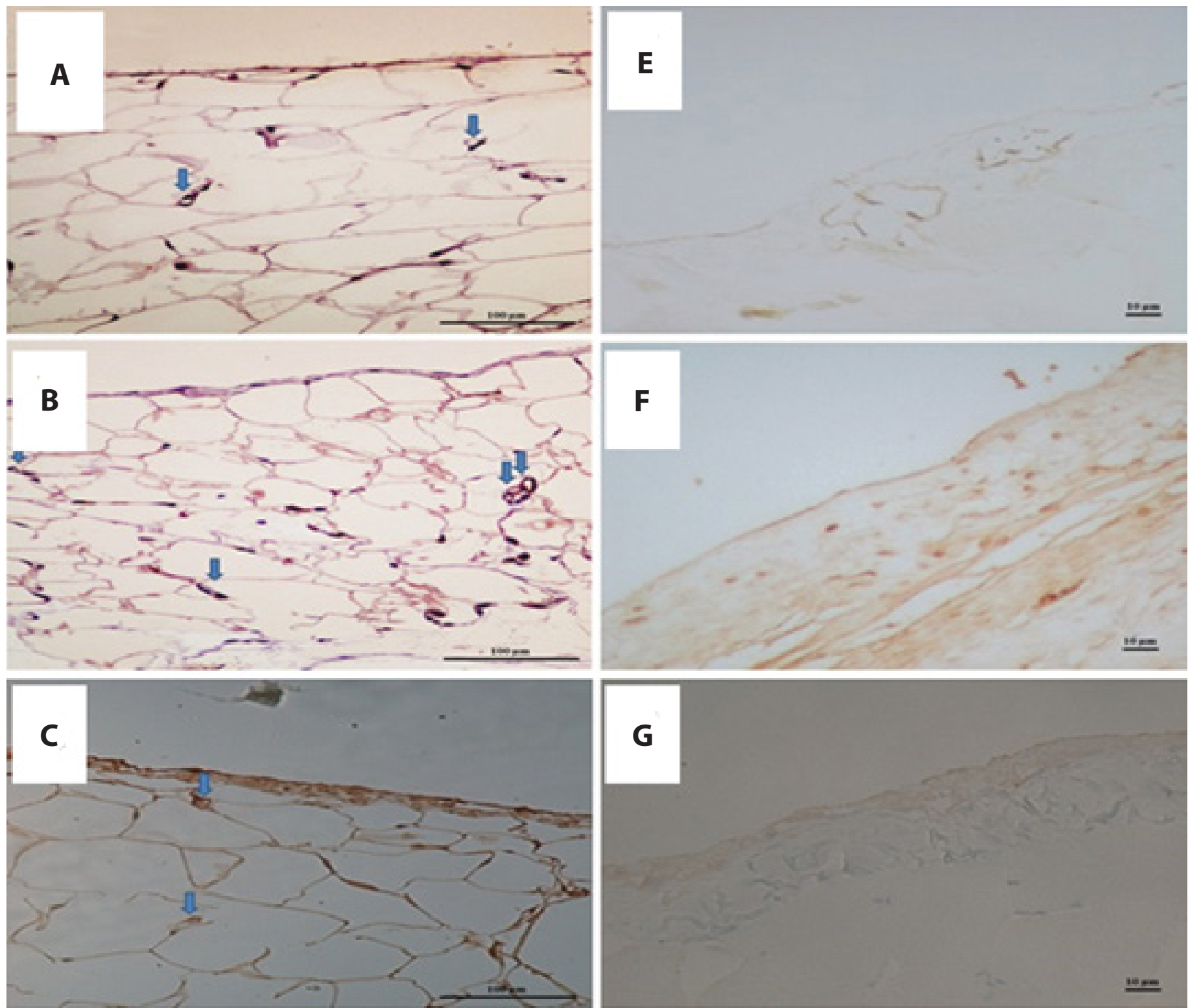

G

Figure 4. The representative figures of vascular density determination by lectin staining (A-C) and hypoxyprobe (E-G) with the average density $(\mathrm{D}$ and $\mathrm{H})$ at 12 weeks after administration of normal saline (NSS, negative control, $\mathrm{A}$ and $\mathrm{E}$ ), peritoneal dialysis fluid (PDF, positive control, $B$ and F), and NSS plus intermittent hypoxia (IH, C and G) were demonstrated (n = 5-6 for $\mathrm{D}$ and $\mathrm{H}$ ). In addition, the representative score of Western blot analysis of HIF-1a from peritoneal membrane (I) from these experimental groups was also indicated (the score was determined from triplicated experiments). ${ }^{*} p<0.05$ value at experimental groups (PDF or IH) vs. NSS, ${ }^{\star} p<0.05$ value at IH vs. PDF 
D



I

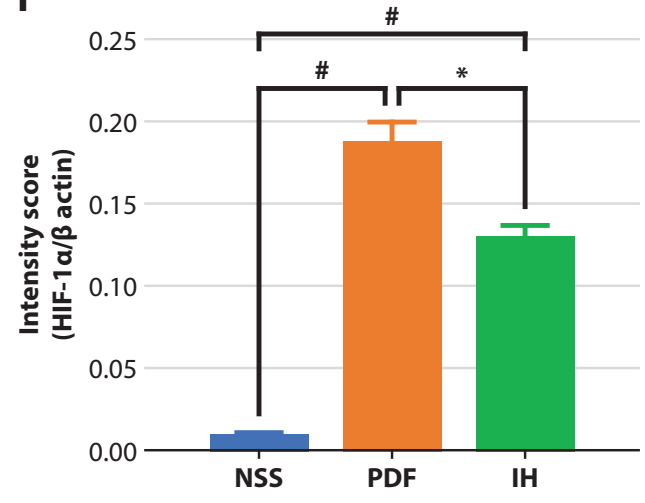

H

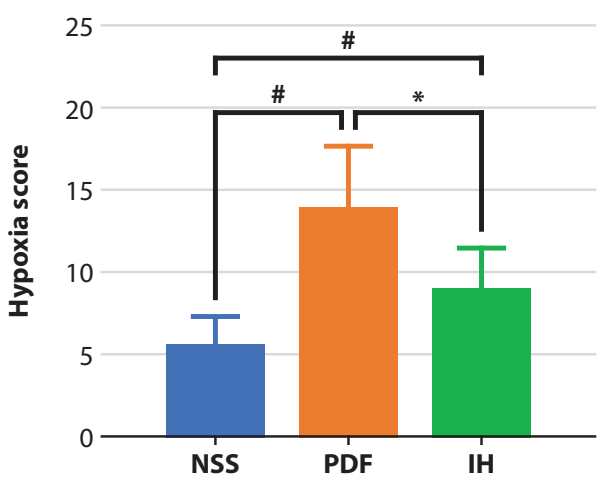

MW marker HIF-1a

\section{$100 \mathrm{LD}$,} .

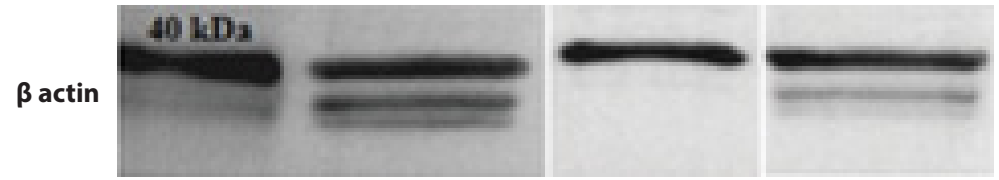

Figure 4. (Continued)

\section{Discussion}

A possible impact of OSA against PD was demonstrated by the thickening of peritoneal membrane in intermittent hypoxic rat model. Intermittent hypoxia induced hypoxic condition in peritoneal membrane which was responsible for the increased HIF-1 $\alpha$ and local pro-inflammatory cytokines in peritoneal membrane. Peritoneal membrane thickening from intermittent hypoxia (a representative of OSA) might be associated with the poor peritoneal function in PD.

Peritoneal membrane is a semi-permeable membrane that is very important for adequate dialysis (PD). ${ }^{24}$ The thickening of peritoneal membrane from several injuries associated with the failure of PD in the disposal of uremic toxins and removal of excessive fluid from patient's body. Indeed, OSA is i) very common, ${ }^{25}$ ii) associated with several metabolic syndrome, ${ }^{26}$ and iii) also enhances renal injury. ${ }^{5}$ Hence, the co-existence of OSA and renal failure is possible. Because several internal organ injuries in OSA are reported through the induction of ROS and cytokines, ${ }^{27-30}$ OSA-induced peritoneal membrane injury is possible. PD fluids injection model was used as a positive control because the continuous peritoneal membrane exposure with a high concentration of glucose in PD fluids-induces cellular hypoxia through glycosylation-associated vascular alteration and PD fluids-induced peritoneal membrane injury is well established. ${ }^{31,14}$

Intermittent hypoxia-induced local cytokine productions in peritoneal membrane with slight elevation of serum cytokines while PD fluids induced only local production of peritoneal cytokines but not systemic cytokines. Intermittent hypoxia, except PD fluids, also induced cytokines in extra-peritoneal sites (liver and kidney) suggesting the systemic injury in intermittent hypoxic model while PD fluids induced only local peritoneal membrane damage. Interestingly, kidney and liver functions (serum creatinine and alanine transaminase) were normal in both intermittent hypoxia and PD fluids, implying that the subtle injury could not be detectable by the routine serum investigation. Because OSA enhanced peritoneal membrane thickening and might worsen the performance of dialysis, routine evaluation of peritoneal membrane such as peritoneal equilibration test (PET) and clearance studies should be recommended in patients with obese. In addition, the protocol for weight reduction in patients with PD and obesity should be recommended as standard care.

Indeed, tissue hypoxia and HIF-1a were detectable in the IH group (more prominent in the PDF group) in comparison with the NSS group supporting the direct injury impact of PD fluids on peritoneal membrane. ${ }^{14}$ Furthermore, the scatter groups of local inflammatory cells in PDF-activated peritoneal membrane with the increased peritoneal membrane thickening and vascular density by PD fluids indicating a more severe inflammatory response compared with intermittent hypoxia model. Nevertheless, hypoxia (hypoxyprobe) induced by intermittent hypoxia, despite less severe than PDF group, was enough to i) activated HIF-1 $\alpha$, one of the critical transcriptional factors responding for cytokine production and angiogenesis, ${ }^{32-34}$ ii) increased peritoneal membrane thickening and iii) enhanced cytokine responses in peritoneal membrane. However, intermittent hypoxia injury was not severe enough to enhance vascular density. Despite a non-different vascular density in the IH group compared with the NSS group, hypoxia (hypoxyprobe) and HIF-1 $\alpha$ were more prominent 
in the IH group implying the limitation of vascular density determination for hypoxia evaluation in patient's histological samples. Likewise, the hypoxia induced expansion of extracellular matrix in peritoneal membrane area in both PDF and IH groups (more prominent in the PDF group) might lead to vascular obliteration and hypoxia of peritoneal membrane as previously mentioned. ${ }^{16}$ However, the combined peritoneal membrane injury with PDF and hypoxia is not performed in this current study. Further study on the combination of intermittent hypoxia plus chronic dialysate administration in the animal model and the influence of obesity in patients with PD are interesting.

\section{Conclusion}

The influence of intermittent hypoxia in OSA might cause the damage on peritoneal membrane, which might be associated with an early peritoneal membrane alteration and PD inadequacy. Hence, further studies on the impact of OSA in patients with PD warrant.

\section{Acknowledgments}

We would like to acknowledge contribution of Jutamas Wongphoom, MSc at tissue processing. This study was supported by the $90^{\text {th }}$ Anniversary of Chulalongkorn University Fund (GCUGR1125594013D), the Thailand Research Fund (MRG-OSMEP505S001), the Government Budget Grant (GRB_10_50_30_80), Rachadaphiseksompot Endorsement Fund (CU-GRS_61_06_30_01), Chulalongkorn University, and National Research Council of Thailand (156/2560). The authors have no conflicts of interest to disclose.

\section{Author contribution}

- WM performed the experiment, collected the data, and drafted the manuscript.

- SK and PR were responsible for pathologic slide preparation.

- SS and KM supervised the experiments.

- TK and AL supervised the study design and edited the manuscript.

- TK raised the funding support.

- $\quad \mathrm{TK}$ and AL are the corresponding authors.

\section{References}

1. Young T, Evans L, Finn L, Palta M. Estimation of the clinically diagnosed proportion of sleep apnea syndrome in middle-aged men and women. Sleep. 1997;20(9):705-6.

2. Parati G, Ochoa JE, Bilo G, Mattaliano P, Salvi P, Kario K, et al. Obstructive sleep apnea syndrome as a cause of resistant hypertension. Hypertens Res. 2014;37(7):601-13.

3. Somers VK, Dyken ME, Clary MP, Abboud FM. Sympathetic neural mechanisms in obstructive sleep apnea. J Clin Invest. 1995;96(4):1897904.

4. Petrosino T, Serafini M. Antioxidant modulation of F2-isoprostanes in humans: a systematic review. Crit Rev Food Sci Nutr. 2014;54(9):1202-21.

5. Abuyassin B, Badran M, Ayas NT, Laher I. Intermittent hypoxia causes histological kidney damage and increases growth factor expression in a mouse model of obstructive sleep apnea. PLoS One. 2018;13(2):e0192084.

6. Gotloib L. Functional structure of the peritoneum as a dialyzing membrane. In: Khanna R, Krediet R, editors. Nolph and Gokal's Textbook of Peritoneal Dialysis. NY, USA: Springer Science+Business Media; 2009. p. 73-102.
7. Floras JS. Sleep Apnea and Cardiovascular Disease: An Enigmatic Risk Factor. Circ Res. 2018;122(12):1741-64.

8. Losso RL, Minhoto GR, Riella MC. Sleep disorders in patients with end-stage renal disease undergoing dialysis: comparison between hemodialysis, continuous ambulatory peritoneal dialysis and automated peritoneal dialysis. Int Urol Nephrol. 2015;47(2):369-75.

9. Garvey JF, Taylor CT, McNicholas WT. Cardiovascular disease in obstructive sleep apnoea syndrome: the role of intermittent hypoxia and inflammation. Eur Respir J. 2009;33(5):1195-205.

10. Snyder B, Shell B, Cunningham JT, Cunningham RL. Chronic intermittent hypoxia induces oxidative stress and inflammation in brain regions associated with early-stage neurodegeneration. Physiol Rep. 2017;5(9): e13258.

11. He Q, Yang QC, Zhou Q, Zhu H, Niu WY, Feng J, et al. Effects of varying degrees of intermittent hypoxia on proinflammatory cytokines and adipokines in rats and 3T3-L1 adipocytes. PLoS One. 2014;9(1):e86326.

12. Williams JD, Craig KJ, Topley N, Von Ruhland C, Fallon M, Newman $\mathrm{GR}$, et al. Morphologic changes in the peritoneal membrane of patients with renal disease. J Am Soc Nephrol. 2002;13(2):470-9.

13. Williams JD, Craig KJ, von Ruhland C, Topley N, Williams GT. The natural course of peritoneal membrane biology during peritoneal dialysis. Kidney Int Suppl. 2003(88):S43-9.

14. Devuyst O, Topley N, Williams JD. Morphological and functional changes in the dialysed peritoneal cavity: impact of more biocompatible solutions. Nephrol Dial Transplant. 2002;17 Suppl 3:12-5.

15. Shimaoka T, Hamada C, Kaneko K, Io H, Sekiguchi Y, Aruga S, et al. Quantitative evaluation and assessment of peritoneal morphologic changes in peritoneal dialysis patients. Nephrol Dial Transplant. 2010; 25(10):3379-85.

16. Sekiguchi Y, Zhang J, Patterson S, Liu L, Hamada C, Tomino Y, et al. Rapamycin inhibits transforming growth factor beta-induced peritoneal angiogenesis by blocking the secondary hypoxic response. J Cell Mol Med. 2012;16(8):1934-45.

17. Li C, Lu J, Zhang B. Development of a novel chronic intermittent hypoxia chamber. Sleep Breath. 2012;16(1):177-9.

18. Nakao A, Nakao K, Takatori Y, Kojo S, Inoue J, Akagi S, et al. Effects of icodextrin peritoneal dialysis solution on the peritoneal membrane in the STZ-induced diabetic rat model with partial nephrectomy. Nephrol Dial Transplant. 2010;25(5):1479-88.

19. Zareie M, Hekking LH, Welten AG, Driesprong BA, Schadee-Eesterman IL, Faict D, et al. Contribution of lactate buffer, glucose and glucose degradation products to peritoneal injury in vivo. Nephrol Dial Transplant. 2003;18(12):2629-37.

20. Rosenberger C, Khamaisi M, Abassi Z, Shilo V, Weksler-Zangen S, Goldfarb $M$, et al. Adaptation to hypoxia in the diabetic rat kidney. Kidney Int. 2008;73(1):34-42.

21. Fisher EM, Khan M, Salisbury R, Kuppusamy P. Noninvasive monitoring of small intestinal oxygen in a rat model of chronic mesenteric ischemia. Cell Biochem Biophys. 2013;67(2):451-9.

22. Carnell DM, Smith RE, Daley FM, Saunders MI, Bentzen SM, Hoskin PJ. An immunohistochemical assessment of hypoxia in prostate carcinoma using pimonidazole: implications for radioresistance. Int J Radiat Oncol Biol Phys. 2006;65(1):91-9.

23. Han WQ, Zhu Q, Hu J, Li PL, Zhang F, Li N. Hypoxia-inducible factor prolyl-hydroxylase-2 mediates transforming growth factor beta 1-induced epithelial-mesenchymal transition in renal tubular cells. Biochim Biophys Acta. 2013;1833(6):1454-62.

24. Breborowicz A, Wisniewska J, Polubinska A, Wieczorowska-Tobis K, Martis L, Oreopoulos DG. Role of peritoneal mesothelial cells and fibroblasts in the synthesis of hyaluronan during peritoneal dialysis. Perit Dial Int. 1998;18(4):382-6.

25. Garvey JF, Pengo MF, Drakatos P, Kent BD. Epidemiological aspects of obstructive sleep apnea. J Thorac Dis. 2015;7(5):920-9.

26. Xu S, Wan Y, Xu M, Ming J, Xing Y, An F, et al. The association between obstructive sleep apnea and metabolic syndrome: a systematic review and meta-analysis. BMC Pulm Med. 2015;15:105.

27. Badran M, Ayas N, Laher I. Cardiovascular complications of sleep apnea role of oxidative stress. Oxid Med Cell Longev. 2014;2014:985258.

28. Muzumdar H, Arens R. Physiological effects of obstructive sleep apnea syndrome in childhood. Respir Physiol Neurobiol. 2013;188(3):370-82.

29. Ciftci TU, Kokturk O, Bukan N, Bilgihan A. The relationship between serum cytokine levels with obesity and obstructive sleep apnea syndrome. Cytokine. 2004;28(2):87-91. 


\section{APJAI}

30. Nadeem R, Molnar J, Madbouly EM, Nida M, Aggarwal S, Sajid H, et al. Serum inflammatory markers in obstructive sleep apnea: a meta-analysis. J Clin Sleep Med. 2013;9(10):1003-12.

31. Sada K, Nishikawa T, Kukidome D, Yoshinaga T, Kajihara N, Sonoda K, et al. Hyperglycemia Induces Cellular Hypoxia through Production of Mitochondrial ROS Followed by Suppression of Aquaporin-1. PLoS One. 2016;11(7):e0158619.

32. Jeong HJ, Chung HS, Lee BR, Kim SJ, Yoo SJ, Hong SH, et al. Expression of proinflammatory cytokines via HIF-1alpha and NF-kappaB activation on desferrioxamine-stimulated HMC-1 cells. Biochem Biophys Res Commun. 2003;306(4):805-11.
33. Tanaka T, Kojima I, Ohse T, Ingelfinger JR, Adler S, Fujita T, et al. Cobalt promotes angiogenesis via hypoxia-inducible factor and protects tubulointerstitium in the remnant kidney model. Lab Invest. 2005; 85(10):1292-307.

34. Ohtomo S, Nangaku M, Izuhara Y, Takizawa S, Strihou CV, Miyata T. Cobalt ameliorates renal injury in an obese, hypertensive type 2 diabetes rat model. Nephrol Dial Transplant. 2008;23(4):1166-72. 